\section{Psychosis in the Elderly}

Edited by Anne Hassett, David Ames \& Edmond Chiu. London:Taylor and Francis. 2005. 276pp. $€ 45.00$ (hb). ISBN I84I843946

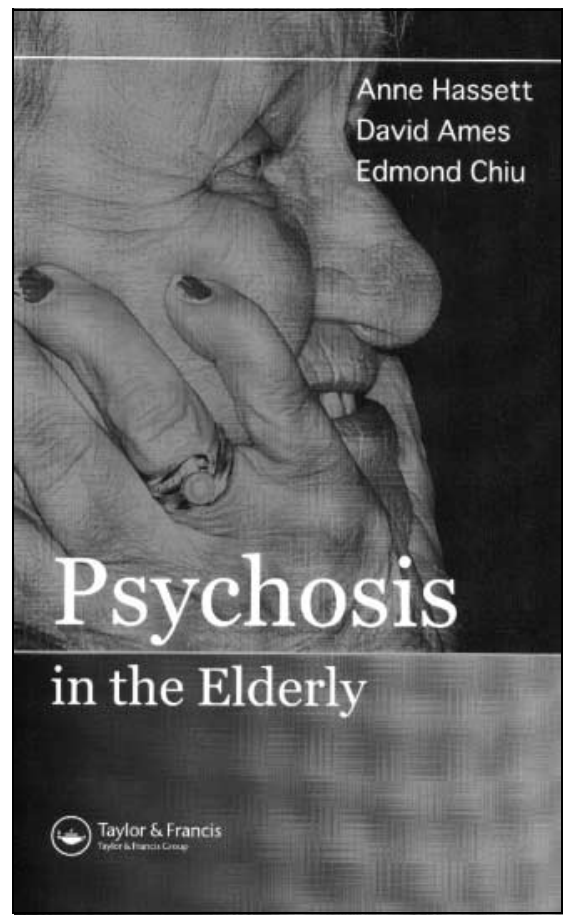

Psychosis in older people receives perplexingly little attention compared with dementia and depression. I have never understood why this should be so, as these patients constitute some of the most memorable that old age psychiatrists encounter and often show remarkable responses to antipsychotic treatment. Opaque and unhelpful terminology, combined with uncertainty about how such patients should be viewed in relation to schizophrenia in young people and psychoses that arise secondary to organic brain conditions, have played important parts in reducing interest. Psychosis in the Elderly is a welcome and accessible multi-author book and an important step in the right direction towards rehabilitation of this area. The title reflects the broadness of scope of the Melbournebased editors' project, which is really a series of excellent mini-reviews on psychosis in association with a wide range of neuropsychiatric disorders as well as schizophrenia in older people. In recent years, some of us have advocated conceptualisation of 'late-onset schizophrenia' as a term to describe cases of schizophrenia with onset delayed beyond the age of 40 and 'very late-onset schizophrenia-like psychosis' for those cases with onset after 60 which seem to have a different set of risk factors and clinical features. Chapter contributors expand on this distinction and update the reader on recent research findings on aetiology, pathophysiology and treatment response. But the most novel aspect of the book is the final section which extends into the territory of psychoses associated with delirium, dementia, affective disorders, stress and alcohol and substance misuse in older people. I would like to see this book sell well and am confident that it will gain deserved recognition as required reading for old age psychiatrists in training.

Robert Howard Professor of Old Age Psychiatry, Institute of Psychiatry, London SE5 8AF, UK. Email: r.howard@iop.kcl.ac.uk

doi: 10.I192/bjp.I89.2.196

\section{The Origins and Course of Common Mental Disorders}

By David Goldberg and lan Goodyer. London: Taylor and Francis. 2005. 248pp. $€ 19.99$ (pb). ISBN I583919600

This book had its first incarnation 12 years ago when Peter Huxley was David Goldberg's co-author. It's just as important a book now as it ever was because of the emphasis on what the community is really suffering from, rather than what the psychiatrist ends up seeing on the wards or in the clinic. The contrast between the two is much wider than most psychiatrists appreciate - much to the frustration of many general practitioners.

But Goldberg and Goodyer marshall current thinking on what really are common mental disorders in a manner that is useful to psychiatrists precisely because they range across viewpoints unfamiliar to many in the profession to bring us a fresh and often surprising perspective on mainstream disorders.

For example, it remains a puzzle why women are more prone to depression than men but one clue many are focusing on is the fact that this profound gender difference seems to emerge after puberty and not before. While it is therefore tempting to locate this corresponding marked and profound contrast in mental health which continues throughout adult life as 'down to hormones', Goldberg and Goodyer suggest here an intriguing theory that will be genuinely novel to most psychiatrists.

The theory locates the centre of the action in terms of mental health in an area of human life which has so far been neglected by psychiatrists - friendship. The first point the authors make is that marked differences begin to emerge in adolescence in the same-sex friendship patterns of boys and girls. First, girls' conversation with friends tends to be more emotional, while that of boys is markedly more reserved. The very emotionality of the language girls use might put more strain on their friendships - in other words they may demand more of their friends than boys do. It may come as no surprise then to learn that girls have a higher turnover of close one-to-one friendships than do boys.

Goldberg and Goodyer make the point that as a result girls are exposed in the longer term to more disappointing experiences than boys within their friendship framework. Perhaps such acute disappointments are potent risks for depression? Perhaps boys are protected from the disappointments of a more intimate and intense social life by their preference for more solitary interests like computing, model-building, collecting stamps or the

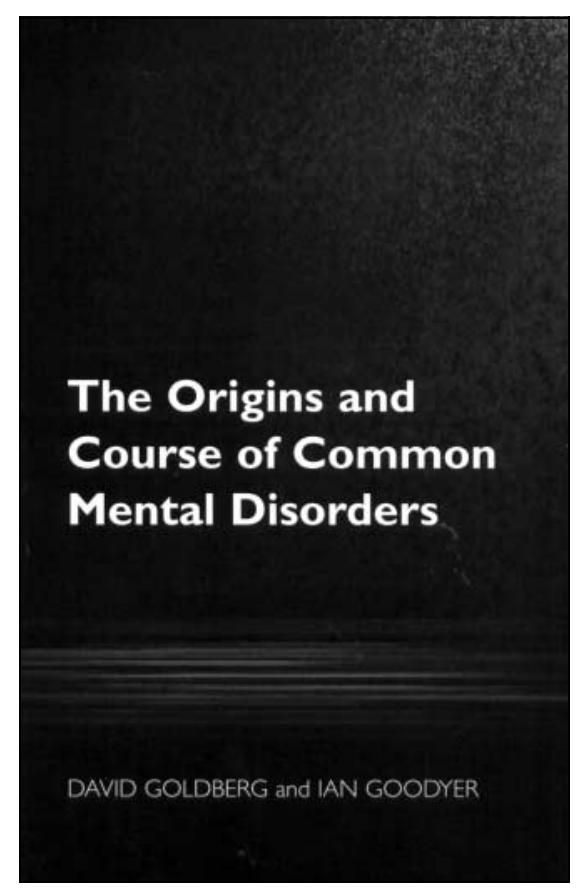

